\title{
INTRODUCTION
}

\section{Y. FUJITA}

Dept. of Astronomy, University of Tokyo, Japan

Stellar infrared spectroscopic survey has advanced in two directions in recent years: in the number of different celestial sources investigated, and in the range of the wavelength region used. It is remarkable that, in the latter case, the scope of the survey is now extending from the photographic infrared region to the far infrared.

When I was asked by the Executive Committee of the IAU to organize the Joint Discussion on 'Stellar Infrared Spectroscopy' in the IAU General Assembly in Sydney, I thought that it would be rather wise and reasonable to restrict it in both directions in order to make our talks more effective and concentrated.

This is the reason why our subjects today are concerned with the study of the nearinfrared region of stars only. Even so, I do hope this meeting may be fruitful with stimulating talks followed by very profitable discussions.

Finally I should like to express my special thanks to each member of the Organizing Committee who has given me many useful suggestions as regards the speakers and the subjects discussed. 